Sonoma Persistent Surveillance System

D. M. Pennington

March 28, 2006 
This document was prepared as an account of work sponsored by an agency of the United States Government. Neither the United States Government nor the University of California nor any of their employees, makes any warranty, express or implied, or assumes any legal liability or responsibility for the accuracy, completeness, or usefulness of any information, apparatus, product, or process disclosed, or represents that its use would not infringe privately owned rights. Reference herein to any specific commercial product, process, or service by trade name, trademark, manufacturer, or otherwise, does not necessarily constitute or imply its endorsement, recommendation, or favoring by the United States Government or the University of California. The views and opinions of authors expressed herein do not necessarily state or reflect those of the United States Government or the University of California, and shall not be used for advertising or product endorsement purposes.

This work was performed under the auspices of the U.S. Department of Energy by University of California, Lawrence Livermore National Laboratory under Contract W-7405-Eng-48. 


\section{Sonoma Persistent Surveillance System}

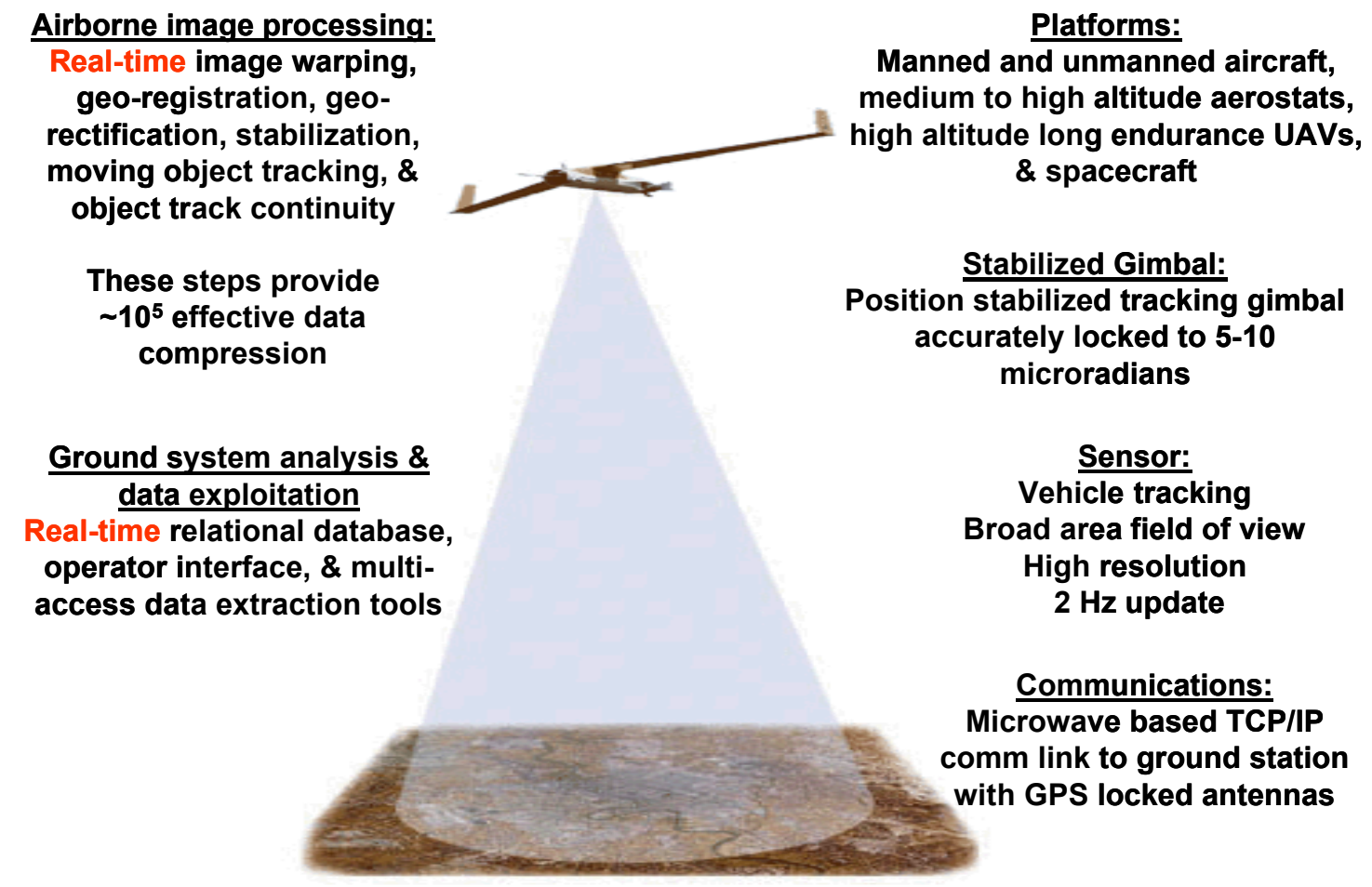

Sonoma offers the first cost-effective, broad-area, high-resolution, real-time motion imagery system for surveillance applications. Sonoma is unique in its ability to provide continuous, realtime video imagery of an area the size of a small city with resolutions sufficient to track 8,000 moving objects in the field of view. At higher resolutions and over smaller areas, Sonoma can even track the movement of individual people. The visual impact of the data available from Sonoma is already causing a paradigm shift in the architecture and operation of other surveillance systems. Sonoma is expected to cost just one-tenth the price of comparably sized sensor systems. Cameras mounted on an airborne platform constantly monitor an area, feeding data to the ground for real-time analysis. Sonoma was designed to provide real-time data for actionable intelligence in situations such as monitoring traffic, special events, border security, and harbors. If a Sonoma system had been available in the aftermath of the Katrina and Rita hurricanes, emergency responders would have had real-time information on roads, water levels, and traffic conditions, perhaps saving many lives. 


\section{Sonoma Persistent Surveillance System}

\section{Submitting Organization: Lawrence Livermore National Laboratory}

Address:

7000 East Avenue, L-183

City:

Livermore

State:

California

Zip code:

94550

Country:

USA

Submitter's Name:

Deanna M. Pennington

Phone:

(925) 423-9234

Fax:

(925) 422-2499

Email:

pennington1@1ln1.gov

AFFIRMATION: I affirm that all information submitted as a part of, or supplemental to, this entry is a fair and accurate representation of this product.

Submitter's signature:

\section{Joint entry: No}

3. Product name: Sonoma Persistent Surveillance System

4. Brief description: Sonoma is an end-to-end systems approach to monitoring a large field of view 24/7 with sufficient resolution and frame rate to track all moving vehicles in the field.

5. When was this product first marketed or made available for order? Sonoma was demonstrated in 2004 and made available to government sponsors and to the private sector for licensing in 2005.

\section{Inventors or Principal Developers \\ Developer Name: $\quad$ Michael Kartz}




\begin{tabular}{|c|c|}
\hline Position: & Systems Engineer/Architect \\
\hline Organization & Lawrence Livermore National Laboratory \\
\hline Address & PO. Box 808, L-183 \\
\hline City & Livermore \\
\hline State & $\mathrm{CA}$ \\
\hline Zip/Postal & 94550 \\
\hline Country & USA \\
\hline Phone & (925) 422-9445 \\
\hline Fax & (925) 422-2499 \\
\hline Email & kartz1@1lnl.gov \\
\hline Developer Name: & Laurence Flath (former LLNL employee) \\
\hline Position: & Chief Technology Officer \\
\hline Organization & Lux Solis, LLC \\
\hline Address & 1214 North P Street, Livermore, CA 94551 \\
\hline City & Livermore \\
\hline State & $\mathrm{CA}$ \\
\hline Zip/Postal & 94551 \\
\hline Country & USA \\
\hline Phone & (925) 487-9735 \\
\hline Fax & (925) 455-1701 \\
\hline Email & lflath@luxsolis.com \\
\hline Developer Name: & Gary Stone \\
\hline Position: & Engineer \\
\hline Organization & Lawrence Livermore National Laboratory \\
\hline Address & PO. Box 808, L-211 \\
\hline City & Livermore \\
\hline State & $\mathrm{CA}$ \\
\hline Zip/Postal & 94550 \\
\hline Country & USA \\
\hline
\end{tabular}




\begin{tabular}{|c|c|}
\hline Phone & (925) 423-0987 \\
\hline Fax & (925) 424-2778 \\
\hline Email & stone4@1lnl.gov \\
\hline Developer Name: & David Bloom \\
\hline Position: & Engineer \\
\hline Organization & Lawrence Livermore National Laboratory \\
\hline Address & PO. Box 808, L-142 \\
\hline City & Livermore \\
\hline State & CA \\
\hline Zip/Postal & 94550 \\
\hline Country & USA \\
\hline Phone & (925) 423-2023 \\
\hline Fax & (925) 423-5846 \\
\hline Email & bloom7@1lnl.gov \\
\hline Developer Name: & Daniel Knight \\
\hline Position: & Software Engineering Consultant \\
\hline Organization & SequoiaTek Corporation \\
\hline Address & 13812 Table Rock Ave. \\
\hline City & Bakersfield \\
\hline State & CA \\
\hline Zip/Postal & 93314 \\
\hline Country & USA \\
\hline Phone & (661) 589-5119 \\
\hline Fax & (661) 589-4553 \\
\hline Email & dknight@sequoiatek.com \\
\hline Developer Name: & John Marion (former LLNL employee) \\
\hline Position: & Director, West Coast Division \\
\hline Organization & Logos Technologies \\
\hline
\end{tabular}


Address

City

State

Zip/Postal

Country

Phone

Fax

Email

Developer Name:

Position:

Organization

Address

City

State

Zip/Postal

Country

Phone

Fax

Email
585 S. L. St.

Livermore

CA

94550

USA

(925) 580-2122

(703) 852-7281

N/A

\section{Robert Sawvel}

Engineering Technical Associate

Lawrence Livermore National Laboratory

PO. Box 808, L-183

Livermore

CA

94550

USA

(925) 422-9620

(925) 422-2499

sawvel3@1lnl.gov

\section{Product price}

LLNL is offering Sonoma as a series of three licenses relating to specific products incorporated into the instrument. The licenses are for:

- The overall system

- The mosaic sensor architecture and associated designs

- Use of the graphics processor-based geo-registration algorithms and visualization software 
Over the last year we have been approach on numerous occasions regarding technology transfer and commercialization of the Sonoma system and technologies, indicating there is a significant commercial market. Several testimonials to the system are included in the Appendix of this submission. Federal business opportunity announcements will be published for each of these three areas in the near future. The price will be determined by the licensees.

\section{Do you hold any patents or patents pending on this product?}

Patent protection is being sought for "Real-Time Geo-Registration of Imagery Using Commercial Off-The-Shelf Graphics Processors" and "A Method for Optically Stitching Multiple Focal Plane Array Sensors to Produce a Larger Effective Sensor with Zero Gaps in the Image Data." Provisional patent filings are currently in effect for both.

\section{Sonoma's Primary Function.}

\section{What does it do?}

The Sonoma system provides a cost-effective means to dramatically improve strategic information gathering and situational awareness by monitoring key sites and regions of strategic importance. Sonoma is capable of continuously monitoring a broad user-selected area, currently the size of a small city, from an airborne platform, producing continuous, real-time, highresolution video imagery. With Sonoma, a user can establish vehicle movement patterns both spatially and over time. This is a key capability for establishing connections between both known and unknown targets, the time history of those connections, and identifying new targets of interest.

We developed the requirements for Sonoma by experimentally collecting and analyzing data over a variety of terrains, urban densities and elevation angles. The resulting system has adequate spatial and temporal resolution to enable of up to 8,000 moving targets within the field of view, and transmission of the data tracks to the ground in real-time. Figure 1 shows the area coverage provided by different sensors superimposed on a satellite image of Washington, DC. A standard CCD camera can provide coverage over a very limited area. HDTV improves the field of view (FOV), but neither camera comes close to covering the area of a city. The current Sonoma sensor 
has 66 Megapixels (Mpx), which enables coverage of the central urban area, with image resolution comparable to the inset shown in the lower right corner. Increasing resolution can be achieved by trading off field of view.

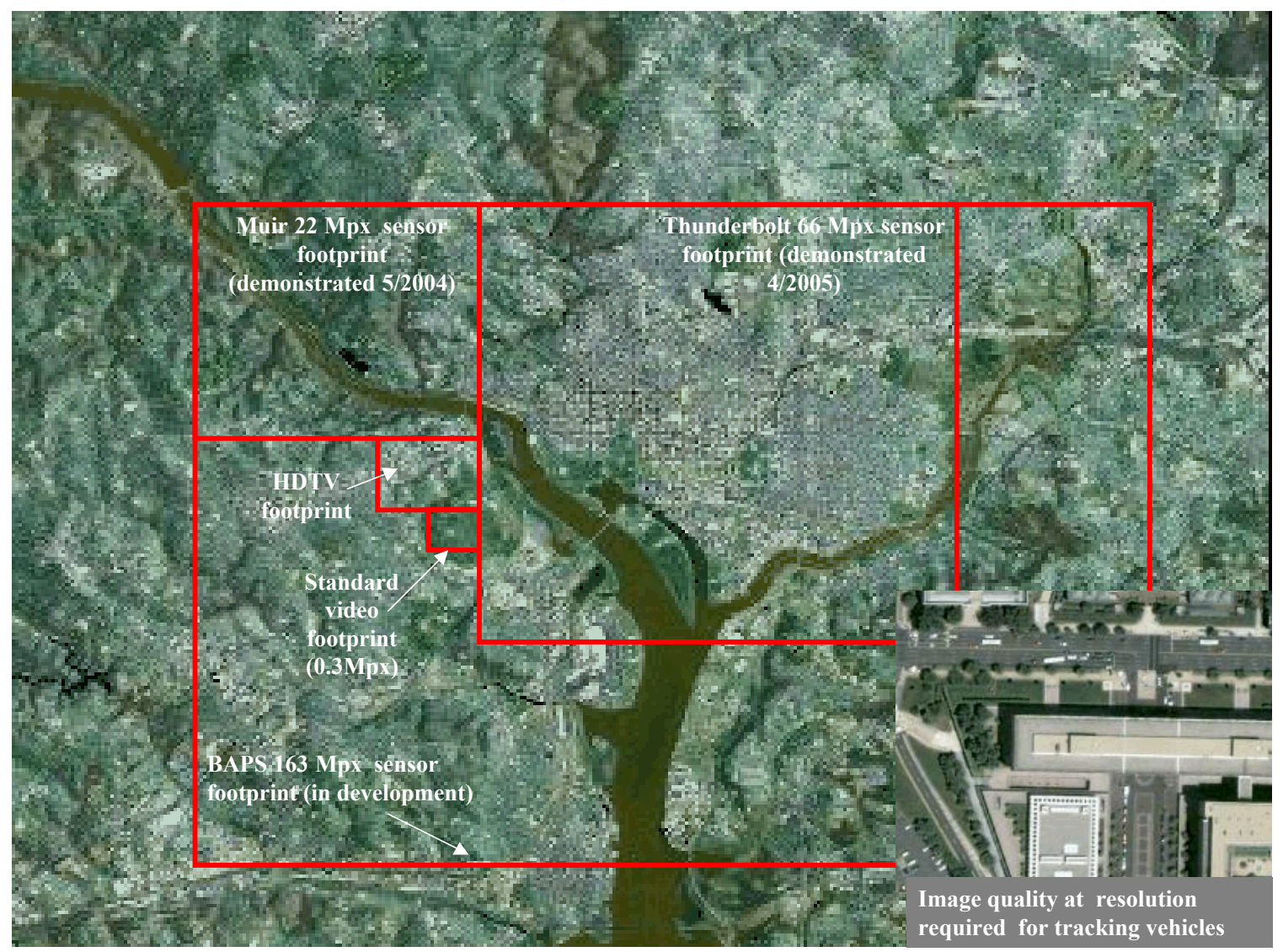

Figure 2. Area coverage provided by a standard video camera, HDTV camera, 22-megapixel (Mpx) sensor (Sonoma prototype 1), and a 66-Mpx Sonoma sensor (Sonoma prototype 2), is indicated by boxes superimposed on a satellite image of Washington, DC. The inset image in the lower right corner is indicative of the resolution provided by the Sonoma sensors with the footprints indicated above.

The main impediment to achieving this capability to date has been the unavailability or cost of three key technologies: affordable persistent platforms, sensors capable of wide area viewing at adequate resolution, and the automated data processing to absorb and analyze the vast data stream from such a system. The Sonoma approach shown in Figure 2 addresses these issues with an end-to-end systems approach, providing innovative solutions that have resulted in significant cost reductions and improved performance. 


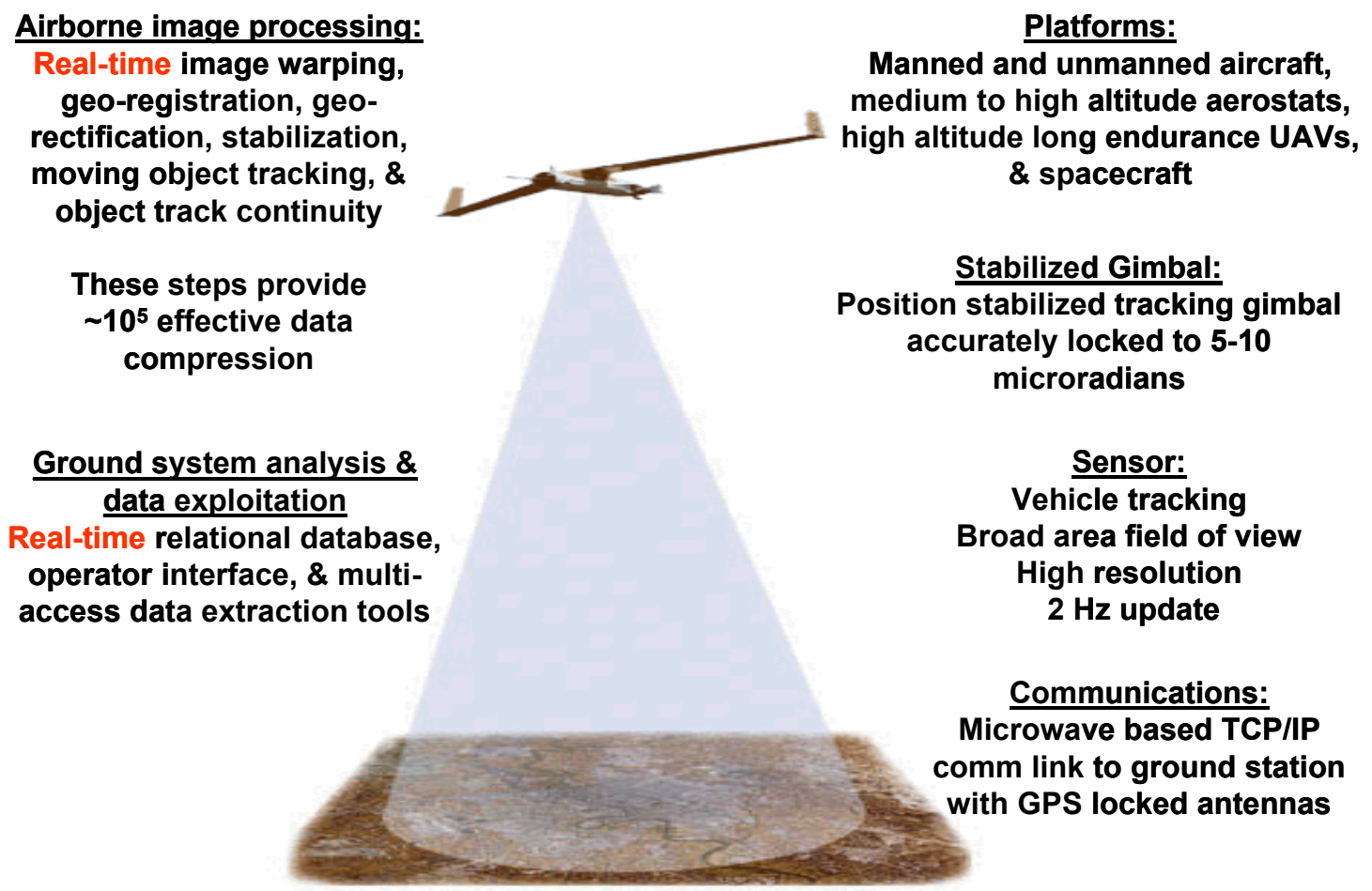

Figure 2. Sonoma is a unique end-to-end systems approach to persistent surveillance over a broad area. The system functions independently of the platform, enabling the use of a broad variety of platforms. Data is collected at a $2-\mathrm{Hz}$ rate for real-time, simultaneous tracking of 8,000 moving objects in the field of view. Sophisticated algorithms, implemented in graphics processors, enable real-time data processing and display. A communications link to a ground station transmits the vehicle track data to the ground in real-time $(2 \mathrm{~Hz})$, which is superimposed on a full video image updated at once per second.

\section{How does it do it?}

The Sonoma system consists of an airborne platform and a ground platform as shown in Figure

2. Each consists of hardware and software integrated together to form a cohesive system that collects, stores, and processes data, allowing an analyst to track both vehicles and people. Onboard image processing consists of real-time geo-registration, a spatial correlation step to stabilize imagery to a single pixel, stabilized background subtraction using GPU-ported algorithms to extract the background from the imagery, detection of moving objects and determination of their centroids, and extraction of the pixels for detected objects. Data transmitted to the ground includes the centroid location of each moving object and an occasional geo-registered image that acts as the map. In future versions, the pixels immediately surrounding each centroid will be transmitted in order to enable object identification. A breakthrough central 
to this development effort was creating the means to perform the geo-registration of these very large data-rate imagery sets in real-time using low-cost, readily available hardware. The use of GPUs for the transformation math (rather than CPUs) is a key to the Sonoma processing efficiency. A detailed discussion of this technology development is given in our papers on "Using Commercial Graphics Processors for Real Time Geo-Registration", 1,2

\section{Sonoma is Scalable}

In an interview published in the July 2002 issue of GIM International, the worldwide Magazine for Geomatics, the then CEO of Z/I Imaging Corporation expressed his belief that "there is nobody coming into the large-format camera market; they cannot afford it. The R\&D is tremendous." Since then, Vexcel produced the first commercially available sensor of comparable size, priced at a cost point of $\sim \$ 800,000$ each (see comparison matrix for details). This sensor stitches multiple apertures together in software to create a composite image, requiring a significant computer capability. To provide a cost-effective alternative, we developed a mosaic stitching technique, for which we are seeking patent protection. The stitching process is accomplished in hardware to provide a large flat FOV. This approach uses commercial off-theshelf (COTS) 11-Mpx, 12-bit cameras, COTS photography lenses, and simple mounting hardware, reducing the cost of the overall sensor to only about $\$ 80,000$ ! All cameras share a common aperture and are focused to infinity, enabling rapid replacement in the field without calibration. The ground sampling distance (GSD) can be changed either by changing the altitude of the airborne platform or by changing out the bayonet-mounted optics for different focal length lenses. The computations required for creating the mosaic image are dramatically reduced by this approach, leaving compute power available for other data processing. This technique is scalable to large aperture arrays in both the visible and infrared spectrum, enabling for the first time a cost-effective solution to large-area focal plane arrays. A $163 \mathrm{Mpx}$ Sonoma sensor prototype is currently under construction.

\footnotetext{
${ }^{1}$ Laurence Flath, "Using Commercial Graphics Processors for Real Time Geo-registration”, Nov. 21, 2003, UCRLPRES-200957

${ }^{2}$ Laurence Flath, Michael Kartz and Randall Frank, "Utilizing Commercial Graphics Processors in the Real-Time Geo-registration of Streaming High Resolution Imagery, Aug. 7, 2004, UCRL-PRES-205737.
} 


\section{Sonoma is Real-Time}

All data taken from the sensor is archived in raw form on the platform for post-flight analysis. However, actionable information requires that an analyst see the data in real-time (i.e., all data processing must be accomplished within the period before the next frame is captured). At two frames per second, the vast data volume generated by our sensor exceeds the bandwidth of all commercial communications links by two orders of magnitude. Standard compression techniques cause artifacts in the data that render automated tracking algorithms unusable. To avoid artifacts and allow the transmission of reasonable volumes of data to the ground, we process the data onboard the airborne platform. A number of software tools have been developed to collect and archive all video data, georegister the data, re-project it to a nadir viewing perspective (data collected at a 30-45 degree elevation angle) and display it in real-time. The regions around each moving object can be transmitted to the ground via an RF communications link in real-time, along with a full video image at a lower rate. Both data types are superimposed and displayed in real-time for the ground-based analyst.

The key to Sonoma's real-time image processing is our specialized software (LLNL's RegRT) combined with standard graphic processors units (GPUs). (We are seeking patent protection for this process.) In a standard computer, GPUs are typically used only 10 percent of the time. However, the $\$ 9$ billion investment in GPU technology by the gaming industry has caused them to increase in speed at twice the pace predicted by Moore's law. GPUs are a natural application specific integrated technology (ASIC) for the image processing required for geo-registration. Use of the GPUs already in all standard computers enables a 100-times acceleration of the georegistration algorithms, so that the imagery from each camera in the mosaic can be processed in real-time. To achieve comparable results on the same time frame, competitors' products require $\sim 30$ CPU Blade processors per camera. The size, weight, power, and heat dissipation required to run the $\mathrm{CPU}$ processors for the full mosaic image make these systems unsuitable for use in unmanned aerial vehicles.

\section{Sonoma Freezes the Background}

Raw imagery collected from a moving platform can be difficult to interpret as the perspective of the imagery changes constantly with the motion of the platform. An example of this can be seen 
in Figure 3(a). Removing this motion and geo-registering the imagery in the traditional manner requires data from three satellites to accurately register the data $(<1$ pixel), or scene-based correlation algorithms, which are computationally intensive and require considerable time. We circumvent this problem by co-bore-sighting a GPS/IMU unit with our sensor, both of which are mounted on a high-precision gimbal (currently a PVLabs XR gimbal). The gimbal is locked on the center of the field of regard to $<10$ microradians, so the center of the image remains stationary as the platform moves. The geo-spatial coordinates and elevation data are recorded directly into the header of each camera frame, enabling the geo-registration and geo-location of each pixel in every frame. Using this information, we geo-register the data in real-time to a flatearth model and re-project it to a nadir viewing perspective. These processes remove the motion of the platform, as seen in Figure 3(b), making the sensor system platform independent. The net result is that the background is frozen, and all moving objects stand out.

The ability to "freeze" the background of a large area from an orbiting aerial platform and to highlight just the moving objects in the scene is at the core of Sonoma's technology. Background subtraction algorithms leave a stationary field showing just the tracks of the moving objects (Figure $3(\mathrm{c}, \mathrm{d})$ ). Since we are primarily interested in moving objects, we save the full-resolution imagery immediately surrounding each moving object and transmit this data to the ground at 2 $\mathrm{Hz}$. The full contextual imagery was also sent to the ground at a rate of once per minute. The resulting reduction in data volume transmitted to the ground is equivalent to $10^{6}$ compression, but without the artifacts. In subsequent post-processing, we have demonstrated the superposition of the tracks on the contextual imagery, shown in Figure 3(e). Traffic in or out of specific regions can be highlighted to identify other locations frequented by each of these vehicles. This feature is not yet available for use in real-time. 


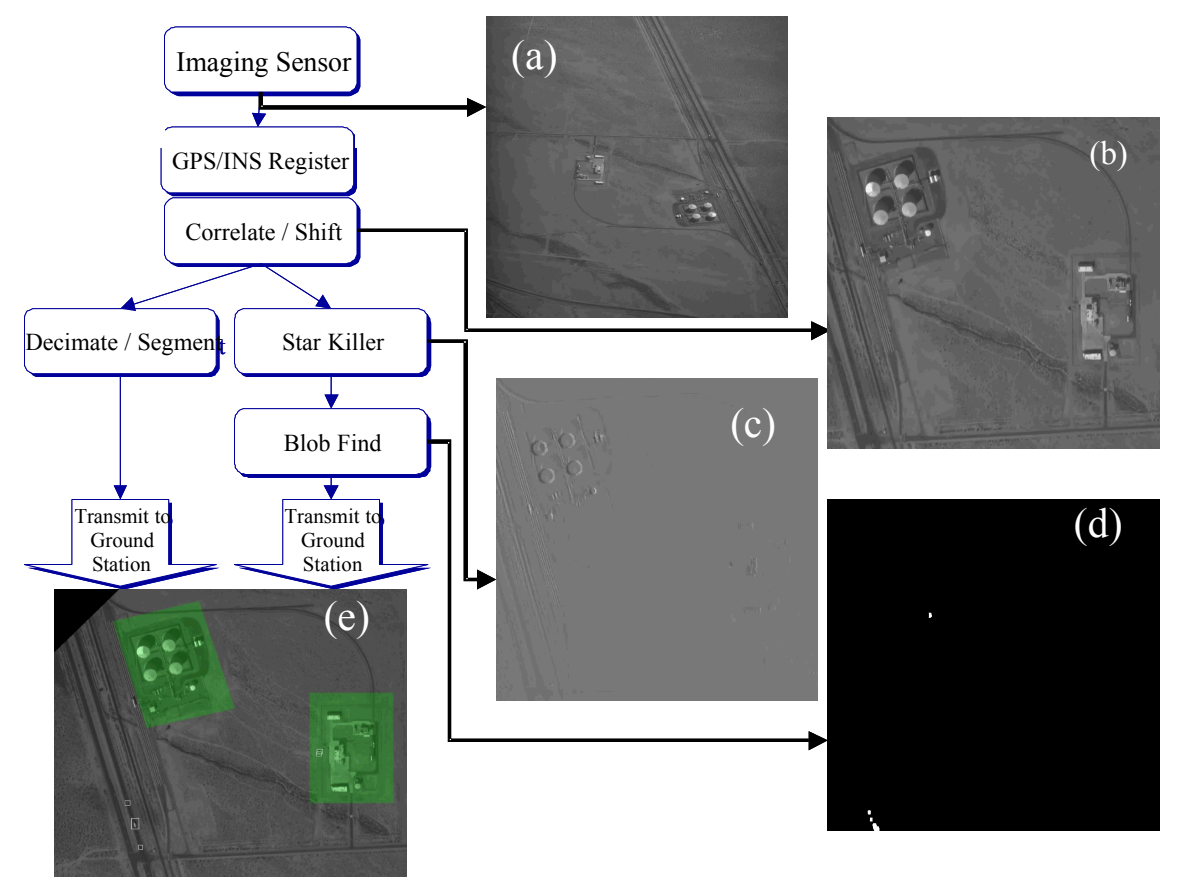

Figure 3. (a) The single-frame raw imagery of a pair of adjacent petrochemical plants near a highway and a railroad track. (b) The geo-registered scene, with the data re-projected as if viewed from nadir (i.e., looking straight down versus the $\mathbf{4 5}$ degrees off-horizon angle from which the data was collected). (c) The subtracted image. A single moving vehicle is visible on the highway near the left edge, and a second vehicle is visible at the right center. Most of the stationary data in the image has been subtracted. (d) The moving vehicles and their tracks are transmitted to the ground in real-time. The full resolution context image is sent down at a slower rate. (e) Post-processed data with movers superimposed on the contextual imagery.

\section{Sonoma Is An Integrated Persistent Surveillance System}

The Sonoma system has been demonstrated in a series of four flight experiments, during which 10 terabytes (TB) of broad-area moving imagery over four different geographical locations were collected. For each data set taken, a coordinated set of ground scenarios (with vehicles and/or people) was executed. Each scenario was designed to evaluate the effectiveness of the Sonoma system. These tests demonstrate all of the key components of the system in action simultaneously, including (a) on-board, real-time data processing, (b) high-bandwidth communications, and (c) a ground station with specialized processing to reconstruct the imagery in real-time. A block diagram of the Sonoma system in shown in Figure 4, with pictures and schematics of the ground station and airborne system in Figures 5 and 6, respectively. 


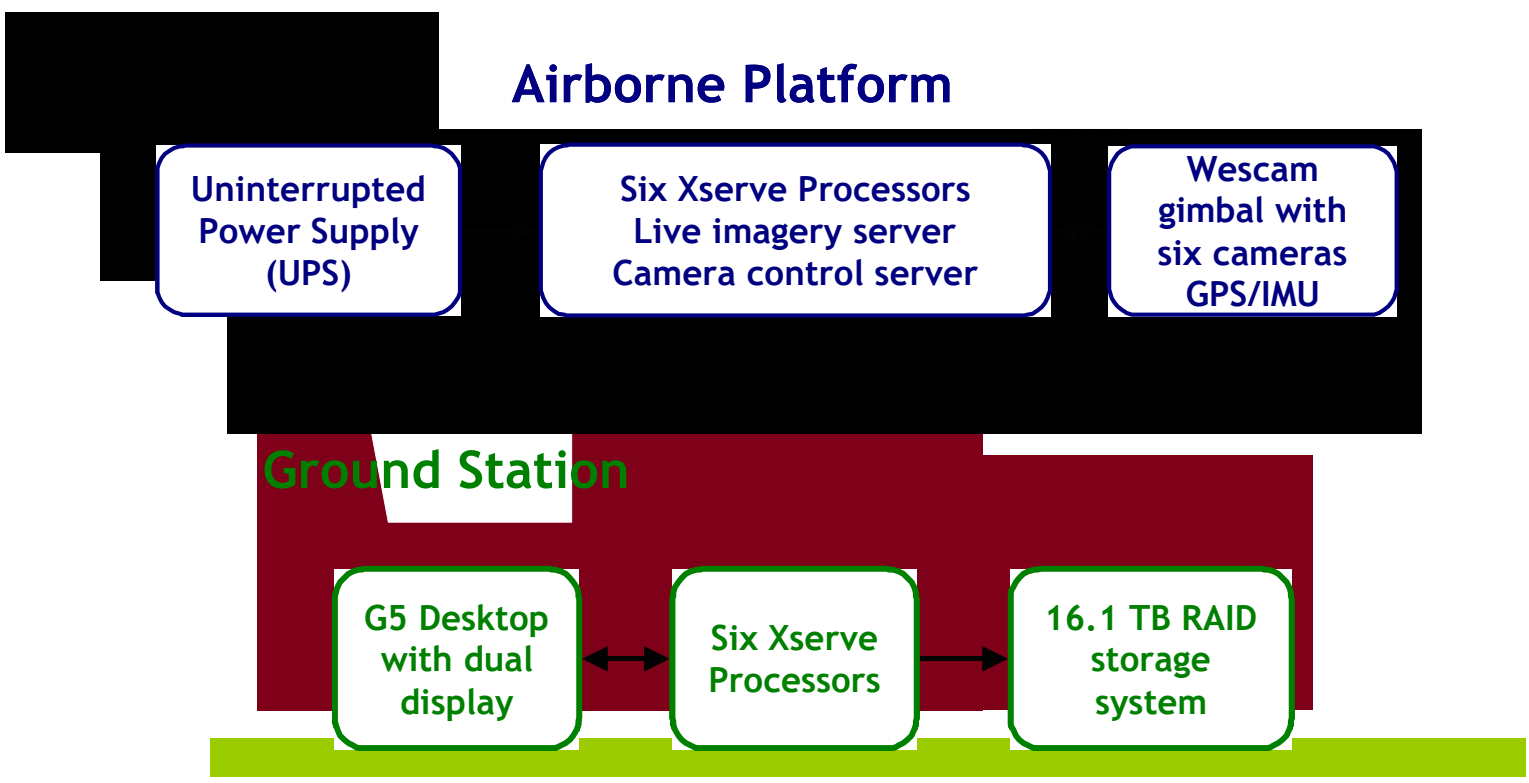

Figure 4. Hardware overview for the Sonoma system.
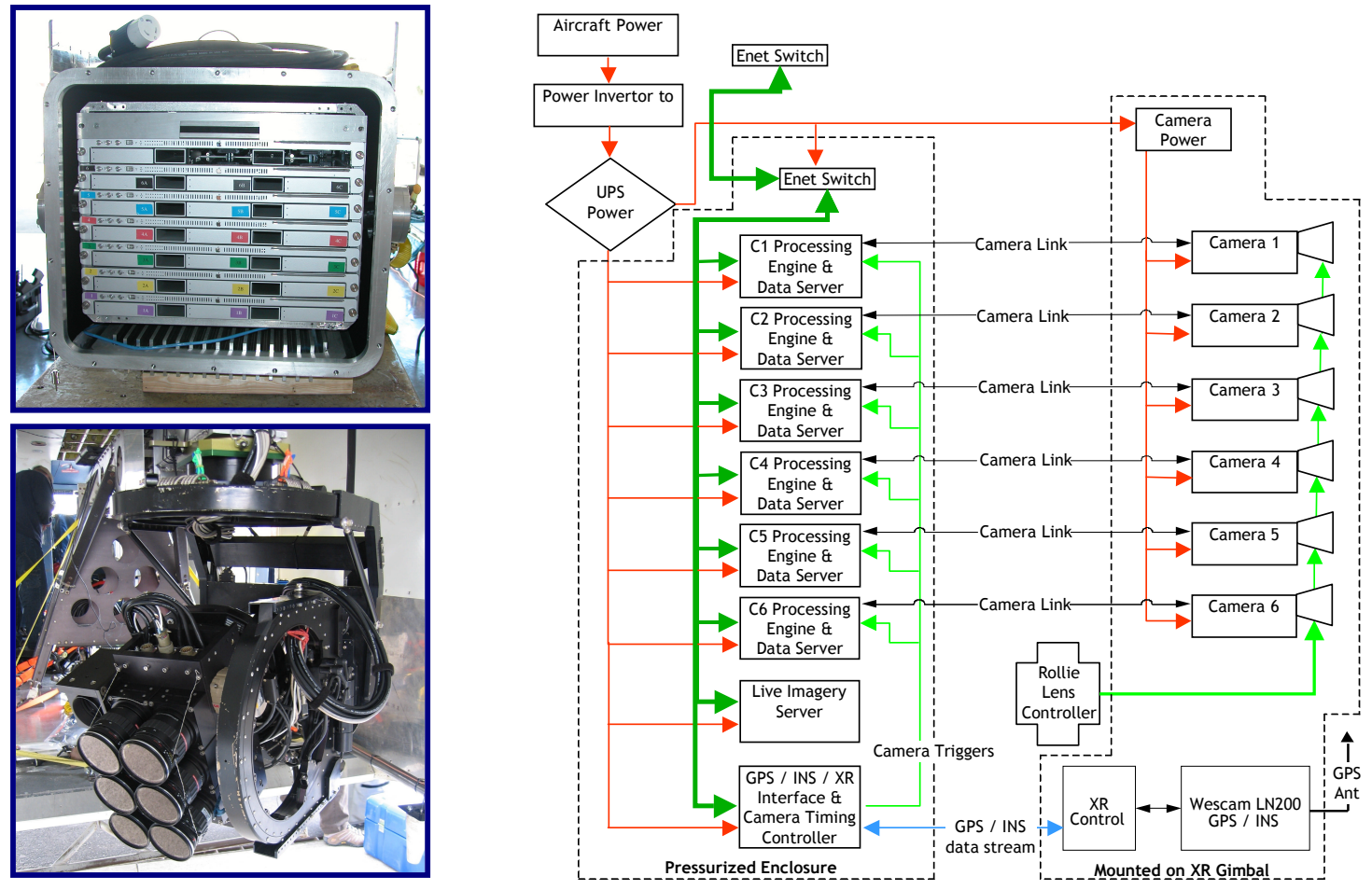

Figure 5. Photos and schematic of the airborne component of the Sonoma system. 

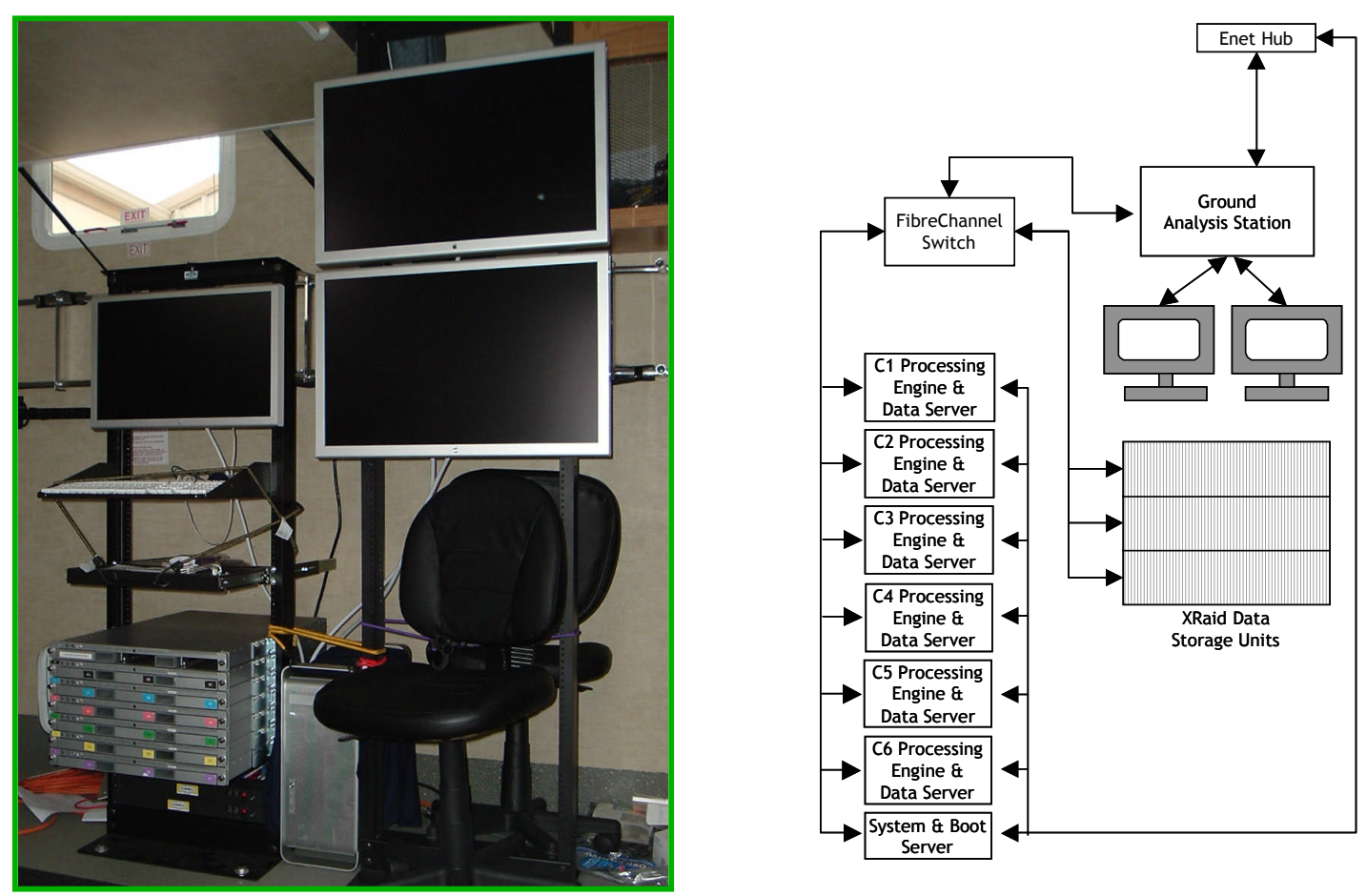

Figure 6. Photo and schematic of the Sonoma ground station.

The flight system is responsible for the acquisition of imagery along with all metadata needed to properly geo-rectify the imagery for post-mission display and analysis. The flight system consists of two main hardware sections: the computer array and the sensor suite. The computer array consists of seven Apple Xserve systems and one Windows XP-powered laptop. Six of the seven Xserves have an Active Silicon frame grabber installed; each of these is cabled to one of the cameras. The top-most Xserve is referred to as the master server and provides the user interface and remote access to all other servers. The laptop provides a trigger for the cameras so they capture images at the same time. The sensor package consists of six $11 \mathrm{Mpx}$ Illunis XMV11000 cameras, a spotter camera, and an LN200 GPS/IMU system mounted in a PV Labs XR gimbal. The camera system is capable of producing a 7504 x 7504 pixel image. Each camera has a $90 \mathrm{~mm}$ Rollei lens attached.

The ground system consists of three sections: an array of 7 Xserve systems, a G5 workstation, and a set of XRAID disks. The Xserve systems are identical to the flight system, except that the master Xserve is not used in the ground system. The G5 workstation replaces the master Xserve as the user interface and remote access to the XServes. It has an NVIDIA 6800 Ultra video card 
installed and is connected to two 30-inch Apple Cinema displays. The visualization software, SonomaView $\mathbb{C}$, makes use of OpenGL protocols and the graphics processing unit (GPU) on the video card to efficiently geo-rectify and display the imagery. Using the GPUs enables a factor of $100 \mathrm{X}$ acceleration of the algorithms used to process the data. Furthermore, the substantial investment in GPUs for the gaming industry is resulting in an increase in performance that by far exceeds Moore's Law for CPUs. The XRAID system is a set of external storage servers used to backup and retain datasets for forensic analysis while the Xserve drives are reused on subsequent flights.

"MuirViewC " is a tool that enables the operator to visualize the aggregate or individual sensor view from the airborne platform. The sensor altitude, viewing angle, lens focal length, pixel size, sensor size, and sensor array size are adjustable variables to help visualize the camera view. The effective pixel size projected onto the ground, along with the footprint, is calculated and displayed. "SonomaViewC" provides the analysis tools for visualizing the image sequences in raw or geo-registered format, which can be played both forward and backwards in time. It gives the operator the capability to visualize the imagery from one individual camera to all cameras in a stitched format, as well as the option to pan through, zoom in and out, and alter the rate at which the imagery is played forward or backward. "SonomaView $\complement$ " also allows the analyst to change the area of interest on a larger scale and dynamically set the effective geo-registered ground resolution. These tools, which are demonstrated on the DVD accompanying this submission, are critical for convenient visualization of the imagery for tracking vehicles. Finally, "CameraSetEditor $C$ " is a tool that builds from a set of individual cameras the aggregate image description XML file to create a unified merged imagery set, allowing the analyst to build a unified ground picture consisting of all, or a subset of, the cameras in the sensor involved in the data collection.

\section{A. Sonoma's Competition.}

\section{Surveillance Systems}

Sonoma is currently the only broad-area electro-optical surveillance system available. There are custom providers for either sensor packages or custom image processing, but Sonoma is unique in offering the only integrated, end-to-end electro-optical surveillance system. Competing 
surveillance systems based on Synthetic Aperture Radar (SAR) can match the resolution of the Sonoma system, though over much smaller fields of view. SAR is an all-weather solution, although the update rate is generally poor and does not meet the requirements for continuously maintaining a lock on moving objects. SAR systems also require 3-point triangulation to georegister imagery.

\section{Electro-Optical Sensors}

The electro-optical system we developed compares favorably to current fielded technology. It has a lower mass, requires less power, and is smaller than radar systems that can track moving targets. However, the performance of the current sensor diminishes at night and the system is not useful in dense cloud conditions. It's low mass allows it to be used on platforms whose endurance is a strong function of payload mass. It also does not have the multipath issues that some radar systems have, and it is primarily used at high elevation angles, which makes it suited for urban tracking. Unmanned platforms are potentially inexpensive enough to produce many of them, allowing use of the system over numerous strategic targets. The sensor is a passive system, compatible with a stealthy platform.

The competing Vexcel sensor (Ultracam 86D) is an 86-Mpx sensor. However, half of the pixels are in the infrared while the other half are in the visible, effectively reducing the field of view to half that of the Sonoma sensor. The frame rate for the Vexcel is slower than the Sonoma sensor (1.3 frames/sec versus 2 frames/sec), making the use of automated tracking algorithms problematic. The Vexcel sensor uses a different approach than Sonoma, in that the cameras are not all pointed at the same location. As a result, the imagery requires significantly more computation to georegister, re-project, and combine than Sonoma's imagery. In addition, the Vexcel design is such that if one camera in the array is incapacitated, it cannot be swapped out with a replacement in the field; thus the entire array must be recalibrated by the manufacturer. Furthermore, the sensor is about ten times more expensive than Sonoma's, and the design does not scale to larger apertures as Sonoma's does. 


\section{Image Processing}

MIT Lincoln Laboratory has a long history of image processing. It has produced an image processing system (not commercially available) and applied it to Sonoma sensor data. The processing engine is capable of most of the same features as Sonoma, including real-time geo registration and data display. The MIT LL viewer also provides automated tracking of vehicles in a user specified $0.25 \mathrm{Mpx}$ section of imagery. Sonoma has demonstrated the real-time transmission of all moving objects within a $22 \mathrm{Mpx}$ section of imagery, and the superposition of those objects on a full resolution $22 \mathrm{Mpx}$ image sent down once a minute. The full real-time 66 Mpx imagery can also be displayed as collected on-board the aircraft, as well as analyzed in ppost-processing. The use of automated algorithms has only been demonstrated in post processing for Sonoma (not real-time). Logos and ITT Industries have also developed imageprocessing engines for use with Sonoma data, though neither have a real-time downlink. In all three cases, our competitors' algorithms operate on standard Blade processors rather than on GPUs. As a result, their systems require up to 180 Blade processors compared to our 6 $\mathrm{CPU} / \mathrm{GPU}$ processors to perform the processing required to display the processed data in realtime. The resulting systems are significantly larger in size and weight than Sonoma, and they require significantly more power and thermal management to operate. This makes the Sonoma GPU processing a more attractive solution for unmanned aerial vehicles, where size, weight, and power needs are key parameters. 


\section{B. Competitive Matrix}

\begin{tabular}{|c|c|c|c|c|}
\hline Comparison Matrix & Sonoma & Vexcel & Raven Eye II & StarSAFire III \\
\hline Incidence angle operation & Nadir to 45 degrees & Nadir & Nadir to 45 degrees & Nadir to 45 degrees \\
\hline Frame Speed & $2-3 \mathrm{fps}$ & $1 \mathrm{fps}$ & $15-30 \mathrm{fps}$ & $15-30 \mathrm{fps}$ \\
\hline Optical Imaging Design & Shared Single Apperature & Multi-Apperature & Single Apperature & Single Apperature \\
\hline Sensor resolution & $\begin{array}{c}66 \mathrm{M}-163 \mathrm{M} \text { pixels } \\
\text { scalable to } 1 \mathrm{G} \text { pixels }\end{array}$ & $82 \mathrm{M}$ pixels & $0.37 \mathrm{M}$ pixels & $16 \mathrm{M}$ pixels \\
\hline Wide FOV & 20-80 degrees & $30-55$ degrees & $0.4-13$ degrees & $0.4-30$ degrees \\
\hline Imagery Storage & Real-Time & Real-Time & Real-Time & Real-Time \\
\hline Persistent Surveillance Capability & Yes - very wide FOV & No & Yes - very small foot print & Yes - very small foot print \\
\hline Persistence Target Lock Accuracy & 2-10 microradians & None & $15-25$ microradians & 15-25 microradians \\
\hline \multicolumn{5}{|l|}{ Processing Features: } \\
\hline Othorectification & Real-Time on platform & Post Analysis & On Ground & On Ground \\
\hline Image Stabilization & Real-Time on platform & Post Analysis & On Ground & On Ground \\
\hline Image Stitching & Opto-Mech none required & Software & On Ground & On Ground \\
\hline Moving Object Detection & 8000 objects in Real-Time & Change detection only & NA & NA \\
\hline Image Processing Engine & $\mathrm{CPU}+\mathrm{GPU}$ & $\mathrm{CPU}$ & $\mathrm{CPU}$ & $\mathrm{CPU}$ \\
\hline Operating system & PC, Mac, or Linux & PC & PC & $\mathrm{PC}$ \\
\hline
\end{tabular}

\section{C. Sonoma versus the Competition.}

Sonoma is unique in its systems approach to the challenges of surveillance. Sonoma improves on competitive technologies by using:

$\circ$ An integrated end-to-end system design.

- A common focal plane array based on commercial off-the-shelf sensors aligned in hardware. This system is significantly lower in cost AND scalable to significantly larger sizes AND reconfigurable and maintainable in the field.

○ Configuration that enables non-nadir viewing.

○ GPU acceleration of algorithms to enable real-time data processing between frames.

- Background subtraction to extract moving objects and reduce the data volume that must be transmitted to the ground station for analysis. 


\section{A. Principal Applications.}

Sonoma was designed specifically for nonproliferation applications. It can track up to 8,000 moving targets in real-time over large areas such that a user can establish vehicle movement patterns both in a spatial and temporal sense. This is a key capability for establishing connections between targets, the time history of those connections, and for identifying new targets. Many issues can be resolved by making connections between facilities, institutions, industrial sites, and specific transportation routes. Tracking facility activity levels, traffic at choke points, and establishing connections between facilities can aid in the understanding of proliferation capabilities and provide guidance for further investigation. Sonoma's real-time processing capability enables the user to act on the information provided by the system in a timely manner. In addition, because all the imagery taken with Sonoma is archived, as is all metadata needed to properly geo-rectify the imagery, the data can be used for forensic analysis following an event.

\section{B. Other Applications.}

Real-time video images and videos have become an increasingly important source of information for remote surveillance, information gathering, situational awareness, and decision-making. With airborne video surveillance, the ability to associate geospatial information with imagery intelligence allows decision-makers to view the geographic context of the situation, track and visualize events as they unfold, and predict possible outcomes as the situation develops. Airborne video surveillance technology is critical to achieving post 9/11 requirements for more ubiquitous and persistent surveillance. In addition, the ability to collect imagery on demand and observe areas of interest for extended periods of time provides great utility for real-time traffic monitoring, special event monitoring, border patrol, harbor security, and emergency response.

\section{Border Patrol}

During initial demonstrations of the Sonoma capability, a number of experiments were performed in collaboration with the U.S. Border Patrol. The system demonstrated the capability to see the movement of vehicles and, at high resolution, even the movement of people.

Deploying Sonoma systems along the border would offer real-time surveillance of traffic across U.S. borders, without the need for manned patrols. Furthermore, airborne deployment provides 
constant surveillance in multiple directions, improving the chance of catching border transgressions.

\section{Maritime Surveillance}

Defense, intelligence, homeland security, customs and immigration, and law enforcement authorities all have a need to monitor vast areas of ocean, coastline, and navigable water in a timely fashion. The fight against terrorism, illegal fishing, alien immigration, piracy, drug trafficking, pollution, and unauthorized cargo shipping all place demands on existing maritime

monitoring systems. Sonoma is a real-time, integrated, end-to-end system that could be used to monitor harbor traffic, detect suspicious activities, and notify harbor authorities to investigate further. Data downlinks from an airborne system could be collected at a ground station that automatically processes this data into imagery products, enabling the user to rapidly detect maritime vessels for subsequent analysis. In addition, unlike conventional satellite imagery, Sonoma is transportable to address problems as they occur in different areas. Our initial demonstrations of the Sonoma capability were performed over San Diego, CA. The data collected clearly demonstrates the ability to discriminate boat traffic in and out of a harbor (see example on the DVD accompanying this submission).

\section{Special Event Monitoring/Law Enforcement}

Law enforcement agencies in large urban areas could use Sonoma to maintain persistent surveillance over a city, a harbor or other waterways, and the movable ground station would enable communications without the interference problems experienced during the events of 9/11 where communications were lost due to the destruction of infrastructure. Orange County is home to nine terrorist threat targets within a small geographical area. Deploying Sonoma above such an area during special events would give law enforcement authorities the ability to monitor for suspicious activities and communicate this information to an operator on the ground in real-time for further investigation. Similarly, having a stationary system deployed over key areas can provide information on potential threats. For example, an aerostat-based Sonoma system over the Washington, D.C. area in 2002 might have identified the activities of the Washington, D.C. sniper, leading to a quicker resolution of the case and fewer deaths. 


\section{Emergency Response}

The use of a Sonoma system in the aftermath of the Katrina and Rita hurricanes could have provided emergency responders with real-time information on the condition of roads, water levels, and traffic conditions. Coordinators could then have rerouted first responders to critical emergencies or identified optimal access routes. The imagery provided by such a system makes possible constant, real-time updates on environmental conditions and responder location. Currently, updates are possible only when a satellite happens to pass over the area.

\section{Traffic Monitoring}

Traffic reporting is a major feature of most news stations in major cities. During rush hour, multiple helicopters patrol over these cities to provide the necessary coverage. A single Sonoma system deployed over an urban area could replace many helicopters, providing automated analysis of the vehicular traffic to the ground in real-time. Emergency vehicles could be dispatched to accidents more quickly. The Department of Transportation has models predicting the expected flow and volume of traffic in many metropolitan areas. But these models must be qualitatively updated. With Sonoma, all traffic in a city could be monitored to validate the models, providing better planning for traffic signals, road construction, and so on.

\section{3-D Dynamic Mapping}

In order to maintain persistent coverage over a defined region, Sonoma's gimbal is pointed to and locked on a specific GPS coordinate, and the airborne platform flies either in a circle or figure eight about that point. Because the Sonoma data is geo-registered in real-time, it can be mapped to a digital terrain map to provide elevation data (future effort). However, in some cases, accurate maps of an area do not exist. Because Sonoma obtains imagery from several perspectives as the platform circles, it has the potential to provide the data necessary to create a digital terrain map of an area in real-time. Such a capability would be invaluable for situational awareness.

\section{Target Identification}


The Sonoma approach allows for not only tracking objects, but for several forms of target identification. For example, the system gets many views of a target from different angles and can infer target dimensions at a level of resolution that exceeds the resolution of the sensor itself. Sun shadows from the multiple directions averaged over many frames can give an indication of vehicle height. Since the Sonoma approach is effectively a video rate, further analysis of the data could reveal parameters such as vehicle velocity, acceleration, vehicle dynamics, and possibly even driver behavior. Velocity and acceleration could be used to estimate vehicle type and loading.

\section{Summary.}

The Sonoma system provides the first cost-effective, broad-area, high-resolution, real-time motion imagery system for surveillance applications. Sonoma is unique in its ability to provide continuous, real-time video imagery of an area the size of a small city with resolutions sufficient to track 8,000 moving objects in the field of view. At higher resolutions and over smaller areas, Sonoma can even track the movement of individual people. Sonoma is expected to cost just onetenth the price of comparably sized sensor systems. While Sonoma was designed to provide realtime data related to proliferation activities, it is also ideally suited for use in situations such as monitoring traffic, special events, border crossings, and harbors. If a Sonoma system had been available in the aftermath of the Katrina and Rita hurricanes, emergency responders would have had real-time information on roads, water levels, and traffic conditions, perhaps saving many lives. The visual impact of the data available from Sonoma is already causing a paradigm shift in the architecture and operation of other surveillance systems.

\section{Contact Person}

Name Yvonne King

Position Partnering Services Administrator

Organization Lawrence Livermore National Laboratory

Address $\quad 7000$ East Avenue

Address L-795

City Livermore

State CA 
Zip/Postal 94550

Country USA

Phone (925) 422-7299

Fax (925) 423-8988

Email_king26@1lnl.gov 


\section{Appendix}

\section{Testimonial Letters:}

1. Scott Reuther, Wave Technologies

2. Nathan Crawford, Consolidated Resource Imaging

3. Michael Meier, ITT Industries

4. Dr. Eiji Yafuso, Lux Solis, LLC

\section{References:}

1) Laurence Flath, "Using Commercial Graphics Processors for Real Time Georegistration", Nov. 21, 2003, UCRL-PRES-200957

2) Laurence Flath, Michael Kartz and Randall Frank, "Utilizing Commercial Graphics Processors in the Real-Time Geo-registration of Streaming High Resolution Imagery, Aug. 7, 2004, UCRL-PRES-205737.

\section{DVD of system and operation}

22 Mpx sensor system deployment

Optical stitching demo

22 Mpx geo-registered San Diego imagery

$66 \mathrm{Mpx}$ sensor system

$66 \mathrm{Mpx}$ reoregistered imagery

SonomaView user interface and analysis 